\title{
Robustness control in bilinear modeling based on maximum correntropy
}

\author{
Valeria Fonseca Diaz ${ }^{1}$ | Bart De Ketelaere ${ }^{1}$ | Ben Aernouts ${ }^{1,2}$ | Wouter Saeys ${ }^{1}$
}

\author{
${ }^{1} \mathrm{MeBioS}, \mathrm{KU}$ Leuven, Kasteelpark \\ Arenberg 30, Leuven, 3000, Belgium \\ E-mail: valeria.fonsecadiaz@kuleuven.be \\ (Valeria Fonseca Diaz), \\ bart.deketelaere@kuleuven.be (Bart De \\ Ketelaere),wouter.saeys@kuleuven.be \\ (Wouter Saeys) \\ ${ }^{2}$ Biosystems TC, KU Leuven, \\ Kleinhoefstraat 4, Geel, 2440, Belgium \\ E-mail: ben.aernouts@kuleuven.be \\ (Ben Aernouts)
}

Funding Information

This research was supported by the FWO Research Foundation Flanders

\begin{abstract}
Summary
We present the development of a bilinear regression model for multivariate calibration based on maximum correntropy criteria (MCC) whose robustness can be easily controlled. MCC regression methods can be more effective when the assumption of normality does not hold or when data are contaminated with outliers. These methods are competitive when the degree of robustness against outliers should be controlled. By controlling the robustness, information from candidate outliers can be partially retained rather than completely included or discarded during calibration. Within the context of bilinear regression models, an MCC approach using SIMPLS is proposed, which is named Maximum correntropy weighted PLS (MCW-PLS). Thanks to the controllable robustness of MCC models, observations are upweighted or downweighted during the calibration process rendering robust models with soft discrimination of samples. Such a weighting represents an important advantage, especially for cases when samples are not drawn from a normal distribution. Applications to three real case studies are presented. These applications uncovered three main features of MCW-PLS: Robustness control between SIMPLS and RSIMPLS, improvements in prediction performance of bilinear calibration models, and the possibility to detect the most informative samples in a calibration set.
\end{abstract}

\section{KEYWORDS:}

maximum correntropy, multivariate calibration, robust models, outliers, normal distribution, sample weights, sample selection

\section{1 | INTRODUCTION}

Within the context of multivariate calibration in spectroscopy, bilinear modeling techniques have been the major research interest for chemometricians because of the optimal trade-off between predictability power and interpretability of regression coefficients ${ }^{1}$. Partial least squares (PLS) has for several decades been the most preferred and used bilinear modeling method for multivariate calibration. The simplest algorithm to solve PLS is known as SIMPLS ${ }^{2}$. It is, however, easily challenged by datasets whose distribution is not normal or unimodal symmetric with light tails $\frac{314 \mid 516}{2}$. Robust approaches, in particular RSIMPLS, were proposed in multivariate calibration to cope with the presence of outliers in the data that badly influence the regression mode ${ }^{5}$. Nonetheless, this robust version of PLS still assumes that the underlying distribution of the data is normal and it aims to detect the outliers that degrade such a distribution in order to discard them from the analysis. Methods for multivariate calibration that can cope with data which do not come from a normal distribution have not been studied extensively ${ }^{7}$. Information theoretic learning (ITL) techniques address this problem and provide a feasible solution to find models that may better fit data that deviate from the normal distribution with a high performance in prediction ${ }^{3 / 8}$. This is achieved by optimizing correntropy criteria instead of minimizing squared errors. From this point of view, 
the concept of a point being an outlier changes towards the amount of information that a sample contains for the statistical model.

Many reports on near-infrared (NIR) spectroscopy applications have commented on the difficulty of gathering reference measurements and on the challenges in controlling multiple sources of variability influencing spectral measurements 9 [10111. Consequently, the reference values and spectral measurements may depict complex probability distributions that cannot be easily described assuming normality in the data. ITL poses an opportunity to find optimal models for complex relationships between spectral values and reference values by measuring similarity based on entropy instead of distance ${ }^{8}$.

MCC versions of principal component analysis were already reported in the literature around 2011 $1^{12}$. Regarding multivariate calibration, NIR applications of MCC linear regression (MCCR) were reported in $2017^{[13}$ showing competitive prediction performance. One practical disadvantage of MCCR is that it does not reduce the dimension of the spectral data into a few components or latent variables, setting it aside of the class of bilinear models and disabling visualization of highdimensional data. This method corresponds specifically to a weighted version of ridge regression (a.k.a Tikhonov regularization) which controls the model complexity through a regularization parameter $\lambda^{13}$. Another MCC approach for multivariate calibration adapts the SIMPLS algorithm to involve a correntropy similarity measure instead of the $L_{2}$-norm similarity measure ${ }^{14}$. In this case, because the MCC modification is based on the covariance matrix, the algorithm generates variable weights instead of sample weights. While variable importance is a relevant feature for the prediction performance of calibration models, the present work focuses on the information weighting of calibration samples in order control outliers or cope with data whose distribution is not normal. In neither of the two works robustness control was found to be taken into account when using MCC methods for multivariate calibration. On the contrary, in the work of $\mathrm{MCCPCA}^{12}$ it is stated that the robustness parameter affects the performance of the models.

In this work, we present a bilinear model to link spectral measurements to reference values by maximizing the correntropy between reference values and low-dimensional scores, rather than minimizing the squared error between these two. The resulting model calculates scores for the samples and provides sample weights in the range $0-1$.

\section{2 | THEORY}

Let $\mathbf{X}_{n \times m}$ be the high dimensional matrix containing spectral measurements for $n$ samples and $m$ variables (wavelengths) and $\mathbf{Y}_{n \times q}$ be the response matrix of reference values. In the following subsections, when it applies, $k$ refers to the number of components or latent variables, $\lambda$ will refer to a regularization parameter and $\rho$ will be the robustness parameter. The main interest of this work concerns finding the best calibration model of the form

$$
\mathbf{Y}=\mathbf{X B}+\varepsilon
$$

\subsection{Least squares}

We refer here to the most used algorithm in multivariate calibration, namely SIMPLS ${ }^{2}$. The high preference for this algorithm is justified because of its computational simplicity, interpretability of regression coefficients and prediction power. Within the framework of data-compression regression models, PLS offers the possibility to represent the high-dimensional data into a space of low dimension, enabling the visualization of the sample space. This is also achieved with Principal Component Regression (PCR) models, but because PLS involves the covariance between regressors and response variables, it usually renders a higher prediction performance for a lower value of $k^{15}$.

Let $\mathbf{S}_{x y}=\mathbf{X}_{c}^{\prime} \mathbf{Y}_{c}$ be the covariance matrix between $\mathbf{X}$ and $\mathbf{Y} . \mathbf{X}_{c}$ and $\mathbf{Y}_{c}$ represent the column-wise centered matrices and $\mathbf{X}_{c}^{\prime}$ represents the transpose of $\mathbf{X}_{c}$. SIMPLS searches for the $k$ orthogonal linear combinations of $\mathbf{X}_{c}$ that maximize $\mathbf{S}_{x y}$ projected onto the low-dimensional space. This criterion takes place in the least squares paradigm, defined by:

$$
\min _{\mathbf{R}}\left\|\mathbf{S}_{x y} \mathbf{S}_{x y}^{\prime}-\mathbf{R}^{\prime} \mathbf{S}_{x y} \mathbf{S}_{x y}^{\prime} \mathbf{R}\right\|^{2}
$$

$\mathbf{R}_{m \times k}$ is known to be the loadings matrix, so that scores in the low-dimensional space can be calculated as $\mathbf{T}_{n \times k}=\mathbf{X}_{c} \hat{\mathbf{R}}$. Upon calculation of the scores, a least squares linear regression model is fitted to link $\mathbf{T}$ to $\mathbf{Y}$ as:

$$
\min _{\mathbf{b}}\left\|\mathbf{Y}_{c}-\mathbf{T b}\right\|^{2}
$$

This way, the final regression coefficients are calculated as $\hat{\mathbf{B}}=\hat{\mathbf{R}} \hat{\mathbf{b}}^{\hat{2}}$. The major challenge for SIMPLS models is their degradation in the presence of outliers ${ }^{5}$.

\subsection{Minimum variance robust estimator}

We refer here to RSIMPLS which is a robust version of SIMPLS $^{4}$. The underlying idea of RSIMPLS is to carry out the SIMPLS approach, but with robust estimators for the mean vector and the covariance matrix for $\mathbf{X}$ and $\mathbf{Y}$. In the case of high-dimensional data, projection pursuit is used on the extended matrix $\mathbf{Z}=(\mathbf{X}, \mathbf{Y})$ to represent the data in a lowdimensional space and find the outliers in it. This process 
uses minimum covariance determinant (MCD) estimation. Initially, the algorithm finds the subset of $h$ observations in the dataset that produce a covariance matrix with minimum determinant. This covariance matrix is then used to flag the outliers of the entire dataset. The final robust covariance matrix and robust mean vector are calculated based on the corresponding inliers 16 . RSIMPLS concludes with a SIMPLS approach applied on the submatrix block $\hat{\boldsymbol{\Sigma}}^{x y}$ of the final robust covariance matrix of $\mathbf{Z}$. The value of $h$ becomes a second tuning parameter in RSIMPLS which, in percentage, can range from $50 \%$ onwards. The lower this value is set, the more robust the model will be 6 .

In order to clarify the use of the $h$ in RSIMPLS, an overview of the algorithm is presented in algorithm 1 In the present work, we refer to the $h$ observations that produce a covariance matrix with minimum determinant as regular observations.

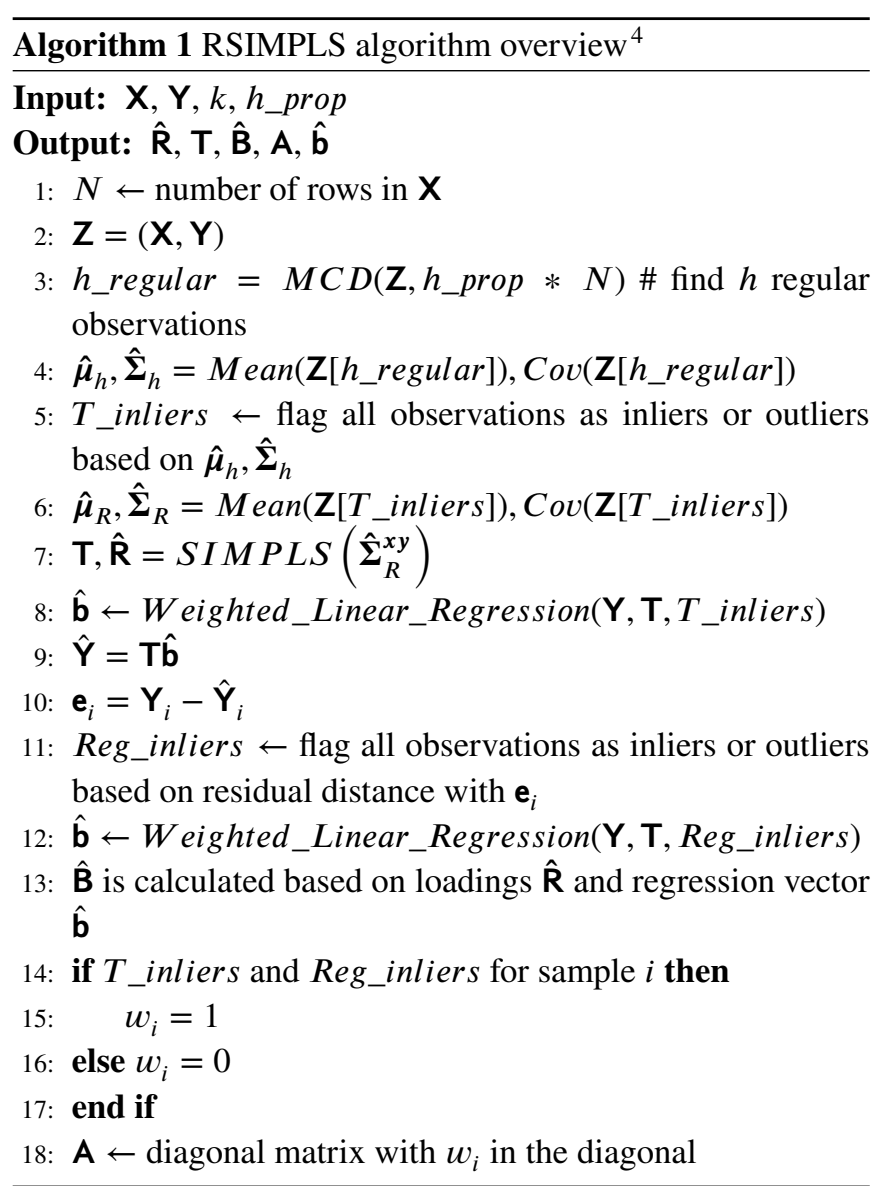

\subsection{Maximum correntropy criterion (MCC)}

To build multivariate calibration models that are robust against outliers or fit better data that are not normally distributed, we use the so-called similarity measure correntropy which involves the inclusion of a robustness control parameter that offers a compromise between least squares and robust methods. Firstly, the paradigm will be introduced, followed by the explanation of the robustness control parameter, current adaptations for multivariate calibration and the proposal of our approach within this paradigm.

\subsection{1 | Definition of the paradigm in MCC}

The paradigm known as maximum correntropy derives from the criterion of minimizing Renyi's quadratic entropy ${ }^{8}$. Let us rely on the generic linear regression model $Y=X \boldsymbol{\beta}+\varepsilon$. Renyi's quadratic entropy of $\varepsilon=Y-X \boldsymbol{\beta}$ is given by:

$H_{2}(\varepsilon)=-\log \left(\int p_{\varepsilon}^{2}(e) d e\right)=-\log (E(p(\varepsilon))=-\log (V(\varepsilon))$

The argument inside the log has been denoted as the Information Potential (IP) of $\varepsilon^{8}$. Keeping the regression parameters $\boldsymbol{\beta}$ unknown, the IP evaluated at $\varepsilon=0$ defines an entropybased similarity measure between $X$ and $Y$ called correntropy (CE). As $p_{\varepsilon}$ is unknown, $\hat{V}(0)$ can be defined by using a nonparametric estimator of $p_{\varepsilon}(0)$ with a Radial Basis Function (RBF) kernel of width $\rho$ as follows

$$
C E(X, Y)=\hat{V}(0)=\hat{p}_{\varepsilon}(0)=\sum_{i=1}^{n} \exp \left(-\frac{\left\|e_{i}\right\|^{2}}{2 \rho^{2}}\right)
$$

where $e_{i}=y_{i}-x_{i}^{\prime} \boldsymbol{\beta}$ and $\left(x_{1}, y_{1}\right), \ldots,\left(x_{n}, y_{n}\right)$ are $n$ observations of a set of i.i.d random variables stored in $X$ and $Y$. Minimizing the entropy $\mathrm{H}_{2}$ is equivalent to maximizing $V$. Therefore, using the correntropy similarity measure $C E$, the regression problem can be solved as follows

$$
\begin{array}{r}
\max _{\boldsymbol{\beta}} C E(X, Y)=\max _{\boldsymbol{\beta}} J(\boldsymbol{\beta})= \\
\max _{\boldsymbol{\beta}} \sum_{i=1}^{n} \exp \left(-\frac{\left\|e_{i}\right\|^{2}}{2 \rho^{2}}\right) \\
\equiv \min _{\boldsymbol{\beta}}-J(\boldsymbol{\beta})
\end{array}
$$

The function $J(\boldsymbol{\beta})$ refers to the similarity measure $C E(X, Y)$ as a function of the parameters of interest $\beta$.

\subsubsection{Solution to the MCC linear regression problem}

The function $J(\boldsymbol{\beta})$ is a non-convex function. In order to find a local minimum for $-J$, half-quadratic optimization (HQ) must be applied ${ }^{12}$. Let us denote the RBF kernel with width $\rho$ as $g(e, \rho)$. There exists a convex function $\phi(e)$ such that 17

$$
-g(e, \rho)=\min _{\alpha}\left(\alpha \frac{\|e\|^{2}}{\rho^{2}}+\phi(-\alpha)\right)
$$


For a fixed $e$, the minimum is reached at $\alpha=g(e, \rho)$. Based on this result, an augmented objective function can be proposed introducing auxiliary parameters $\boldsymbol{\alpha}=\left(\alpha_{i}\right)_{i=1}^{n}$ given by

$$
L(\boldsymbol{\beta}, \boldsymbol{\alpha})=\sum_{i=1}^{n} \alpha_{i}\left(\frac{\left\|y_{i}-x_{i}^{\prime} \boldsymbol{\beta}\right\|^{2}}{2 \rho^{2}}\right)+\phi\left(-\alpha_{i}\right)
$$

Given this augmented objective function, the optimization problem can be defined as

$$
\min _{\boldsymbol{\beta}}-J(\boldsymbol{\beta})=\min _{\boldsymbol{\beta}, \boldsymbol{\alpha}} L(\boldsymbol{\beta}, \boldsymbol{\alpha})
$$

Thus, minimizing $-J(\boldsymbol{\beta})$ is equivalent to minimizing the augmented objective function $L(\boldsymbol{\beta}, \boldsymbol{\alpha})$. According to the previous result, for a fixed $\hat{\beta}$, it holds true that ${ }^{17}$

$$
-J(\hat{\boldsymbol{\beta}})=\min _{\boldsymbol{\alpha}} L(\hat{\boldsymbol{\beta}}, \boldsymbol{\alpha})
$$

with solutions

$$
\hat{\alpha}_{i}=\exp \left(-\frac{\left\|y_{i}-x_{i}^{\prime} \hat{\boldsymbol{\beta}}\right\|^{2}}{2 \rho^{2}}\right)
$$

Similarly, for a fixed $\hat{\boldsymbol{\alpha}}$, the problem becomes

$$
\min _{\boldsymbol{\beta}} \sum_{i=1}^{n} \hat{\alpha}_{i}\left(\frac{\left\|y_{i}-x_{i}^{\prime} \boldsymbol{\beta}\right\|^{2}}{2 \rho^{2}}\right)
$$

which corresponds to a weighted least squares problem with observation weights

$$
a_{i}=\hat{\alpha}_{i} / 2 \rho^{2}
$$

The solution is therefore found at

$$
\hat{\boldsymbol{\beta}}=\left(X^{\prime} \mathbf{A} X\right)^{-1} X^{\prime} \mathbf{A} Y
$$

where $\mathbf{A}_{n \times n}$ is a diagonal matrix with weights $a_{i}$ on the main diagonal. By iterating equations (13) and (16), the solution converges to a local minimum 17 .

\subsection{3 | Robustness control in MCC}

As seen in the previous section, the inclusion of a nonparametric estimator for the density $p_{\varepsilon}(e)$ involves a new tuning parameter which corresponds to the RBF kernel width $\rho$. Maximum correntropy methods are classified within the domain of robust methods in regression ${ }^{3}$. The actual robustness in MCC is controlled by the kernel width $\rho$. As the RBF kernel measures an exponential similarity between $X$ and $Y$, such a similarity can be relaxed or shrunk as $\rho$ becomes larger or smaller. Although not exactly the same, MCC methods with a large value of $\rho$ may resemble least squares solutions ${ }^{3}$. In what follows, MCC methods for multivariate calibration will be discussed together with our proposal for the model in equation (11).

\subsection{4 | Maximum correntropy methods for multivariate calibration}

Maximum correntropy methods have been given attention in several applications involving image recognition and dimension reduction ${ }^{17 / 12}$. In particular, two MCC approaches for multivariate calibration have been proposed. The first one consists in applying MCC regression with a modification of the solution in equation $(16)$ as follows 13

$$
\hat{\mathbf{B}}=\left(\mathbf{X}_{c}^{\prime} \mathbf{A} \mathbf{X}_{c}+\lambda \mathbf{I}\right)^{-1} \mathbf{X}_{c}^{\prime} \mathbf{A} \mathbf{Y}_{c}
$$

where I is the identity matrix of order $m$. The complexity of the model is controlled by the regularization parameter $\lambda$. We refer to this regression method here as MCCR. Note that setting the sample weights matrix $\mathbf{A}$ to the identity matrix in equation (17) renders ridge regression $\frac{18}{18}$. According to Peng et al. (2017), the kernel size can be calculated as:

$$
\rho^{2}=\frac{1}{n} \sum_{i=1}^{n} e_{i}^{2}
$$

The second approach consists in using the correntropy similarity measure for the difference in equation (2) developing the so-called MCCPLS ${ }^{14}$. MCCPLS follows an equivalent development proposed for MCC Principal Component Analysis by Ran, H. 2011 12 . By doing so, MCCPLS implies the calculation of variable weights instead of sample weights ${ }^{14}$. While this can be interesting from the perspective of variable selection, it does not provide the robustness control we were aiming for in this study.

\subsection{5 | Maximum correntropy weighted PLS (MCW-PLS)}

In order to maintain the discrete dimension reduction of $\mathbf{X}$ while performing MCCR to control the robustness of calibration models, we present an approach that takes sample weights from an MCCR step to solve a weighted SIMPLS step. We named this method Maximum correntropy weighted PLS (MCW-PLS).

Following the least squares approach for classical SIMPLS as in equation (2), weighted SIMPLS can be formulated as

$$
\min _{\mathbf{R}}\left\|\mathbf{S}_{w} \mathbf{S}_{w}^{\prime}-\mathbf{R}^{\prime} \mathbf{S}_{w} \mathbf{S}_{w}^{\prime} \mathbf{R}\right\|^{2}
$$

where $\mathbf{S}_{w}=\mathbf{X}_{c}^{\prime} \mathbf{A} \mathbf{Y}_{c}$ is the weighted covariance matrix with sample weights stored in the diagonal matrix $\mathbf{A}$. With loadings matrix $\hat{\mathbf{R}}$, scores for samples are defined as in SIMPLS. These scores are to be used within an MCCR framework as 


$$
\min _{\mathbf{b}} \sum_{i=1}^{n} \exp \left(-\frac{\left\|\mathbf{Y}_{c(i)}-\mathbf{t}_{i}^{\prime} \mathbf{b}\right\| \|^{2}}{2 \rho^{2}}\right)
$$

which is solved as explained in section 2.3.2. Therefore, the sample weights can be taken from the resulting $\alpha_{i}$ weights as in equation (15) to construct the diagonal matrix $\mathbf{A}$ and solve the weighted SIMPLS problem in (19). The process is to be repeated for several iterations or until convergence. Note that the mean vector for $\mathbf{X}$ and $\mathbf{Y}$ is also recalculated by using the updated sample weights. The pseudo-code of MCW-PLS is presented in algorithms 2 and 3

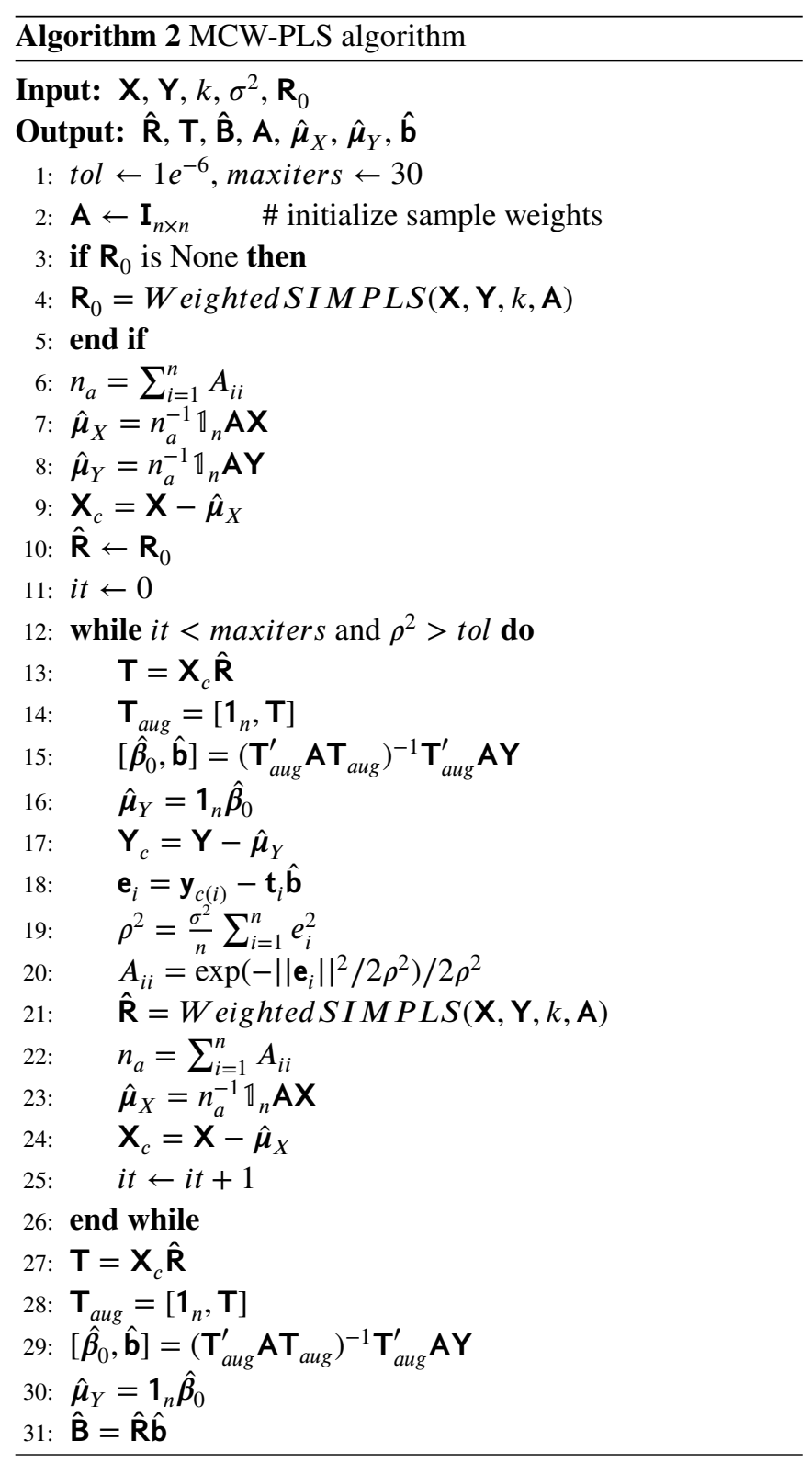

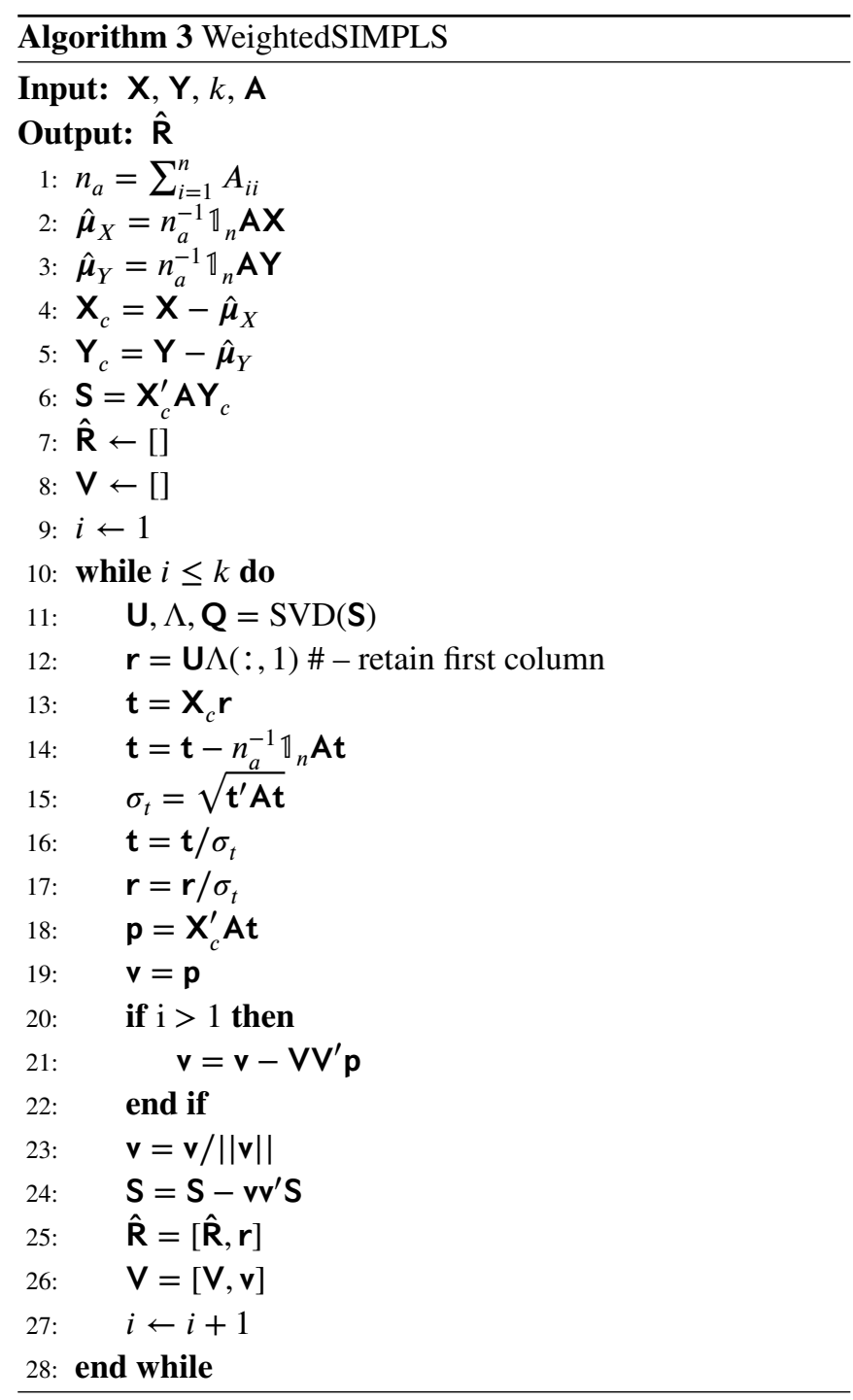

Motivated by the proposal for MCCPCA in Ran et al. (2011) $)^{12]}$ and MCCR in Peng et al. 2017 13 , the kernel size is defined in MCW-PLS as

$$
\rho^{2}=\frac{\sigma^{2}}{n} \sum_{i=1}^{n} e_{i}^{2}
$$

where $\sigma^{2}$ represents the factor by which the mean squared error is multiplied in each iteration ${ }^{12}$. This $\sigma^{2}$ becomes the robustness tuning parameter in MCW-PLS.

\section{MCW-PLS Notation}

Algorithms 2 and 3 compose the entire procedure in MCWPLS. $\mathbf{X}$ refers to the spectral matrix of size $n \times m$ and $\mathrm{Y}$ refers to reference measurements in a matrix of size $n \times q$. The number 
of latent variables is represented by $k$ and $\sigma^{2}$ corresponds to the robustness parameter factor. Matrix $\mathbf{R}$ is of size $m \times k$ and contains the loadings to calculate the latent variables. Matrix A is a diagonal matrix of size $n \times n$ containing the sample weights on the diagonal. This matrix is initialized by the identity matrix $\mathbf{I}_{n \times n}$. To calculate weighted means when it holds, matrices $\mathbb{1}_{n}$ of size $n \times n$ and $1_{n}$ of size $n \times 1$ were used. The latter contain 1's in all their entries. The resulting regression coefficients are: matrix $\mathbf{B}$ of size $m \times q$, intercept coefficients $\hat{\boldsymbol{\beta}}_{0}$ of size $1 \times q$ and slope coefficients $\mathbf{b}$ of size $k \times q$.

\section{3 | EXPERIMENTAL}

\section{1 | Case studies}

The first case study corresponds to the Octane public dataset containing 39 samples in total ${ }^{4}$. The NIR spectra of these gasoline samples were measured at 226 wavelengths in the range $1100-1550 \mathrm{~nm}$ together with reference values for the octane number of the samples. Out of the 39 samples, 6 are known to contain an addition of ethanol ${ }^{5}$. These special samples are referred to as spectral outliers and the rest are denoted regular observations. This case study was used to position MCW-PLS with respect to SIMPLS and RSIMPLS. A test set of 5 samples was selected with a Kennard-Stone algorithm ${ }^{19}$ ensuring that the spectral outliers were kept in the calibration set.

The second case study corresponds to the Corn public dataset ${ }^{14}$, containing 80 samples whose NIR spectra were measured at 700 wavelengths in the 1100-2500 $\mathrm{nm}$ range and whose reference values are available for moisture, oil protein and starch. The 80 samples were divided into 64 calibration and 16 test samples. To select the test samples, a ROBPCA ${ }^{6}$ was performed to select the inlying observations, followed by the selection of 16 samples with a Kennard-Stone algorithm 19 on this subset. This partition strategy ensured that potential outliers remained in the calibration set to test the robustness control of calibration models.

The third case corresponds to a study on milk composition. The data was collected and analyzed by Aernouts et al. $(2011)^{9}$. It consists of 300 samples measured for reflectance and transmittance in the range 350-2500 $\mathrm{nm}$ with resolution of $1 \mathrm{~nm}$ and reference values for fat, protein and lactose. The 300 samples were divided into 240 calibration samples and 60 test samples with the same strategy as in the second case study.

\subsection{1 | Preprocessing}

Mean centering of spectral variables and response variables for the three case studies was applied. Note that in MCW-PLS, MCCR and RSIMPLS the mean vectors are weighted averages depending on the resulting calibration sample weights. For the

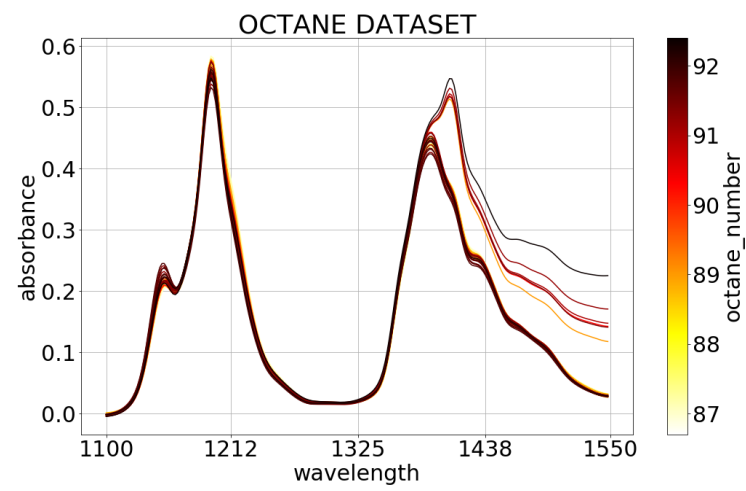

FIGURE 1 Octane dataset spectra

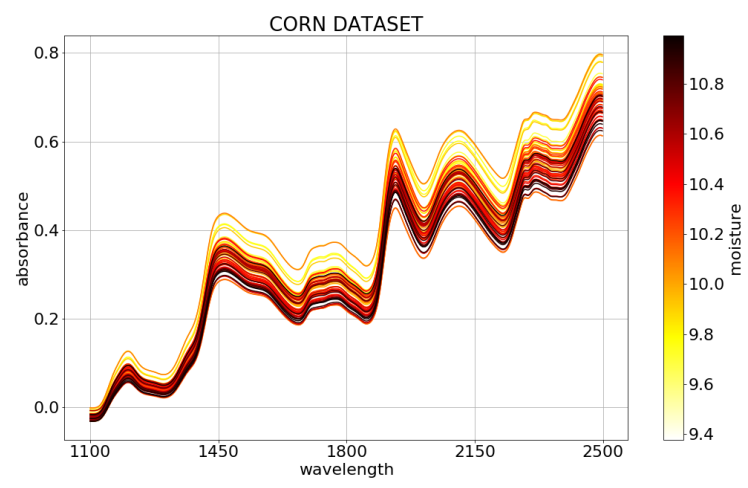

FIGURE 2 Corn dataset spectra

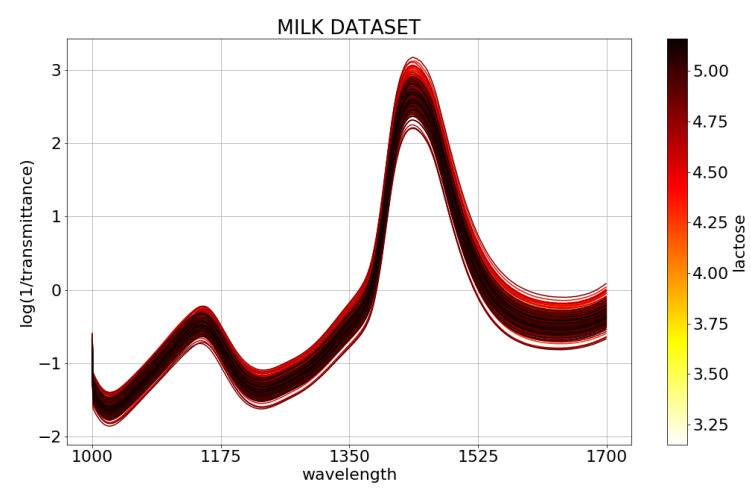

FIGURE 3 Milk dataset spectra

third case study, the wavelength range was set to the interval $1000 \mathrm{~nm}-1700 \mathrm{~nm}$ as it was done by Aernouts et al. (2011)

\subsection{Data analyses}

We demonstrate the positioning and performance of MCWPLS with respect to SIMPLS and RSIMPLS and compare the prediction performance with MCCR. SIMPLS, MCWPLS and MCCR were programmed in Python as scikit-learn 
classes 20 . The LIBRA toolbox available for Matlab under academic licenses was used for RSIMPLS21.

\subsection{1 | Model performance evaluation}

In all the subsequent analyses, the model performance was evaluated based on root mean squared error (RMSE) and coefficient of determination $\left(R^{2}\right)$. These values were calculated for cross-validation (RMSECV, $R^{2} \mathrm{CV}$ ) and prediction (RMSEP, $R^{2} \mathrm{P}$ ). In the case of RMSECV, a robust calculation was adopted in which the weights of calibration samples are included as follows

$$
R M S E C V=\sqrt{\frac{1}{\sum_{i=1}^{n} w_{i}} \sum_{i=1}^{n} w_{i}\left\|\mathbf{Y}_{i}-\hat{\mathbf{Y}}_{i}\right\|^{2}}
$$

where the value of $w_{i}$ depends on the algorithm. For SIMPLS, it corresponds to 1, for RSIMPLS $w_{i}=0$ or $w_{i}=1$ as depicted in algorithm $1^{122}$. For MCW-PLS and MCCR, $w_{i}=a_{i}$ as depicted in algorithm 2 and equation (13). The RMSEP was calculated analogously to equation (22) for test samples where all $w_{i}=1$.

Similarly, the $R^{2} \mathrm{CV}$ was calculated as the coefficient of determination in the weighted linear regression between $Y$ and $\hat{\mathrm{Y}}$. The $R^{2} \mathrm{P}$ was also calculated in this way for test samples where all $w_{i}=1$.

\subsection{2 | Model selection tuning parameters}

For SIMPLS, RSIMPLS and MCW-PLS, the number of latent variables was tuned in a range from 1 to maximum 20. For MCW-PLS, besides the number of latent variables, the robustness factor $\sigma^{2}$ was tuned. During the experiments MCW-PLS provided very similar results to SIMPLS for values of $\sigma^{2}$ larger than 4 , therefore this factor was tuned in a range from values close to 0 up to maximum 4 . For RSIMPLS, the number of latent variables and the percentage of regular observations were tuned. The latter was tuned in a range from $50 \%$ to $90 \% \frac{16}{16}$. In addition, the regularization parameter $\lambda$ for MCCR was tuned for values between 0 and 1 . In all cases, RMSECV curves were calculated and visualized for model selection. For MCCR, MCW-PLS and RSIMPLS, models were selected based on the global minimum RMSECV across ranges of their corresponding tuning parameters. If the same minimum value was observed at different numbers of latent variables for SIMPLS, MCW-PLS and RSIMPLS, the lowest of these numbers was chosen. The selected number of latent variables in MCWPLS was chosen in SIMPLS in the case where its RMSECV curves had a decreasing behavior.

\section{4 | RESULTS AND DISCUSSION}

\subsection{Octane dataset}

The public dataset Octane was used to demonstrate the theoretical positioning and investigate the prediction performance of the MCW-PLS algorithm. The spectra of this dataset are shown in Figure 1 Because of the clear separation of the 6 samples with extra alcohol from the rest of the samples, this dataset has been used in different applications regarding robust methods for multivariate calibration 6 .

Figure 4 shows the RMSECV curves for the three bilinear algorithms. The curves in MCW-PLS are colored by the robustness parameter $\sigma^{2}$ and in RSIMPLS by the percentage of regular observations. In SIMPLS, the RMSECV curve had a long constant tail from 3 latent variables onwards. In MCW-PLS, for $\sigma^{2}$ close to 0.001 , the RMSECV minimum was rendered at 4 or 6 latent variables. For larger values of $\sigma^{2}$, the RMSECV minimum was observed at 3 or 6 latent variables. As for RSIMPLS, setting the regular observations close to $50 \%$ rendered an RMSECV minimum at 3 latent variables. When this percentage was set close to $80 \%$, the minimum was found at 4 latent variables. For larger percentages, the minimum was reached at a larger number of latent variables. SIMPLS was set at 3 latent variables. We selected 3 models for RSIMPLS to show the performance for different percentages of regular observations. Likewise, after inspection of MCW-PLS models across the range of $\sigma^{2}$, we selected 3 models that rendered a different model performance than SIMPLS and RSIMPLS and could explain the transition in robustness. Finally, MCCR reached a minimum RMSECV at $\lambda=0.0013$. The performance of these selected models is shown in Table 1 It can be seen that the MCW-PLS model with 3 latent variables and $\sigma^{2}=0.1$ rendered the lowest RMSECV and RMSEP.

Figure 5 (a) shows the resulting scores plot in the first 2 latent variables based on SIMPLS. It can be clearly seen that the first component was highly influenced by the spectral outliers, describing the variability with respect to this group of samples. The second latent variable took control on the variability of regular observations. The opposite result is observed in the scores plot of RSIMPLS (Figure 5(b)). The transition from this non-robust result to a complete robust result rendered by RSIMPLS was achieved by MCW-PLS when decreasing the value of $\sigma^{2}$. Figure 6 shows the redirection of the latent variables as $\sigma^{2}$ took values $0.1,0.03$, and 0.001 . The resulting model with the highest value of $\sigma^{2}$ highly resembled the latent variables rendered by SIMPLS. For the second value of $\sigma^{2}$, a slight change in the position of the points on the score plot was produced. Finally, when $\sigma^{2}$ was lowered more, the resulting latent variables resembled the result of the RSIMPLS model, as shown in Figure 6 (c). In this final step, both MCW-PLS and RSIMPLS downweighted the spectral outliers to 0. However, 


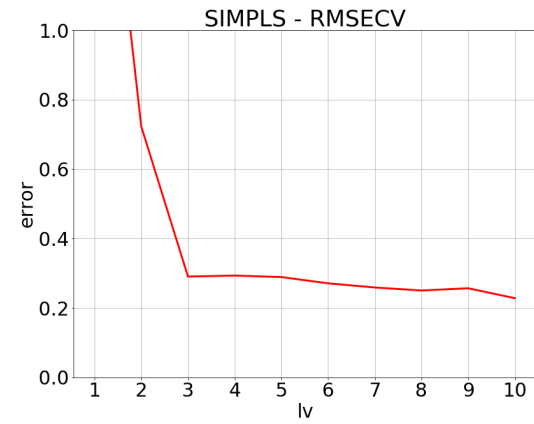

(a)

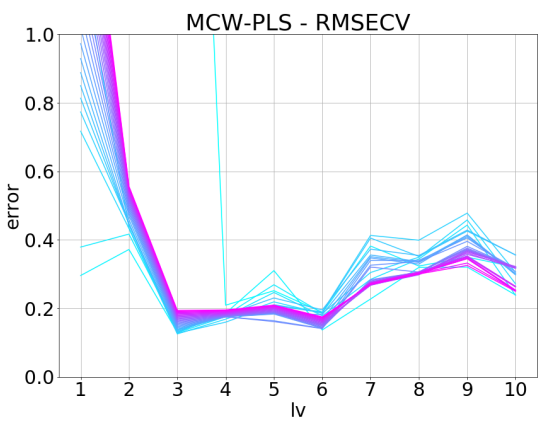

(b)

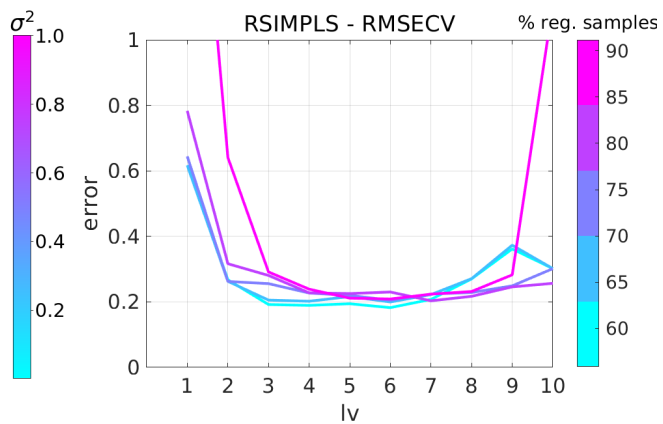

(c)

FIGURE 4 RMSECV curves Octane dataset

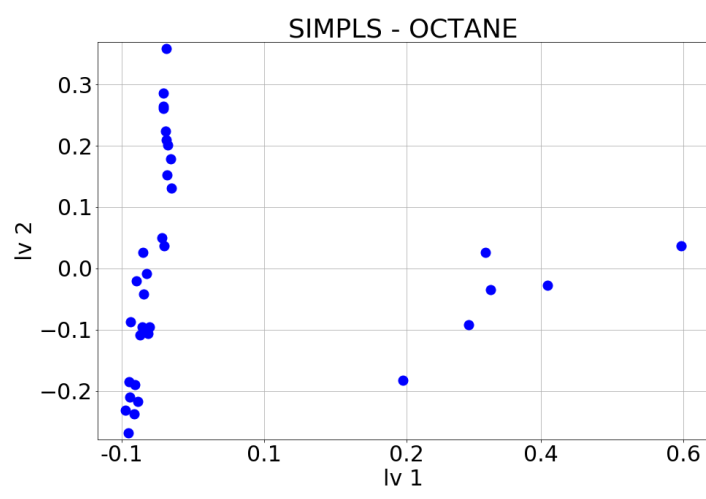

(a) SIMPLS Model 1 in Table 1

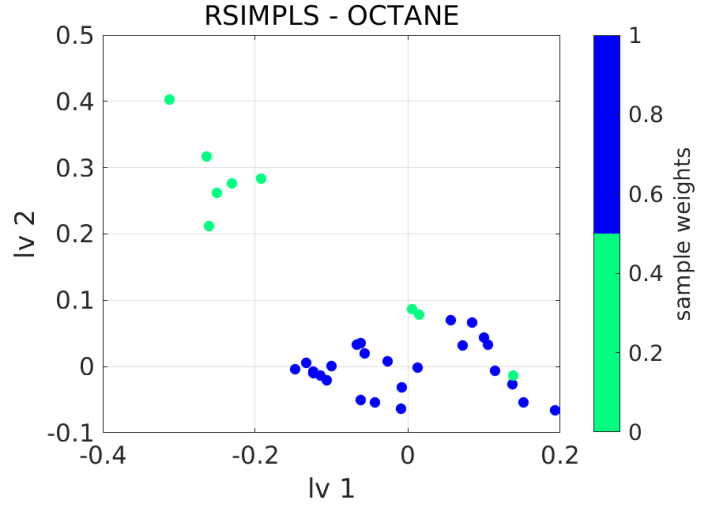

(b) RSIMPLS Model 3 in Table 1

FIGURE 5 SIMPLS and RSIMPLS scores plot of Octane dataset

when comparing the prediction performance with the degree of robustness of the model, the best MCW-PLS model suggested that 5 of the 6 spectral outliers were informative samples for the calibration model (Figure 6(a)).

\subsection{Corn dataset}

The Corn public dataset was used here to study the prediction performance of the different models. For this application, the prediction of moisture content was considered. The spectra of this dataset are shown in Figure 2. A thorough description of

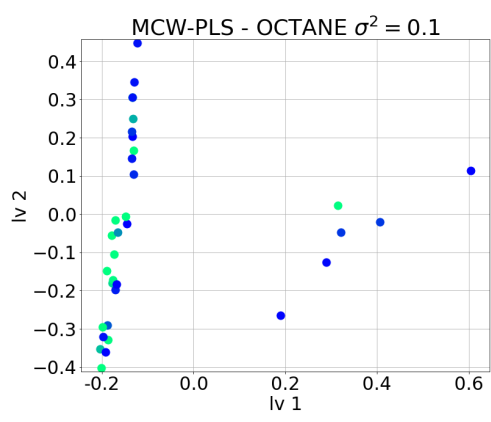

(a)
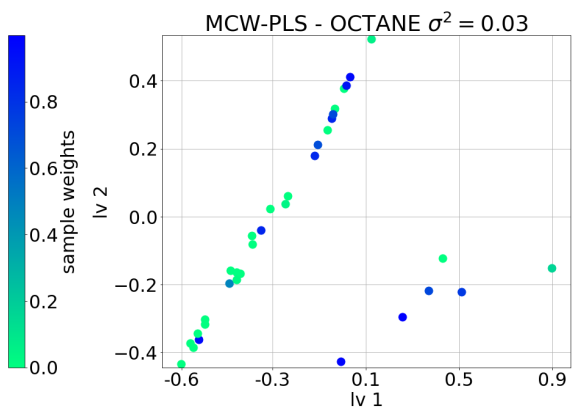

(b)
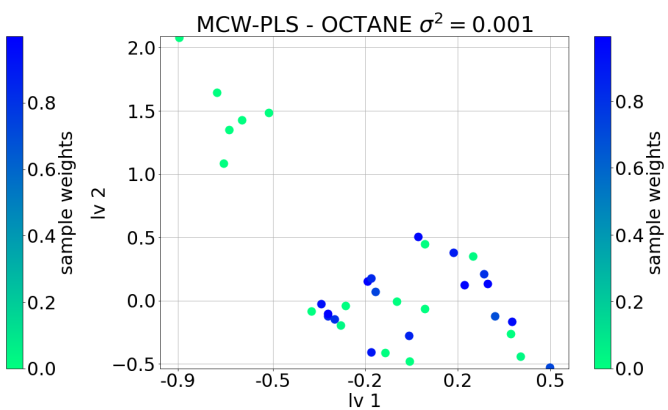

(c)

FIGURE 6 MCW-PLS scores plot of Octane dataset 


\begin{tabular}{|l|c|c|c|c|c|c|c|c|c|}
\hline \multicolumn{1}{|c|}{ Algorithm } & Model & $\lambda$ & lv & $\%$ reg. samples & $\sigma^{2}$ & RMSECV & R2CV & RMSEP & R2P \\
\hline SIMPLS & 1 & & 3 & & & 0.290 & 0.978 & 0.238 & 0.986 \\
\hline \multirow{3}{*}{ MCW-PLS } & 1 & & 4 & & 0.001 & 0.208 & 0.989 & 0.281 & 0.978 \\
& 2 & & 3 & & 0.03 & 0.134 & 0.995 & 0.291 & 0.989 \\
& 3 & & 3 & & 0.1 & 0.130 & 0.996 & 0.184 & 0.989 \\
\hline \multirow{3}{*}{ RSIMPLS } & 1 & & 3 & $56 \%$ & & 0.192 & 0.995 & 0.327 & 0.971 \\
& 2 & & 2 & $71 \%$ & & 0.262 & 0.982 & 0.277 & 0.982 \\
MCCR & 3 & & 4 & $79 \%$ & & 0.228 & 0.992 & 0.256 & 0.988 \\
\hline
\end{tabular}

TABLE 1 Selected models for Octane dataset

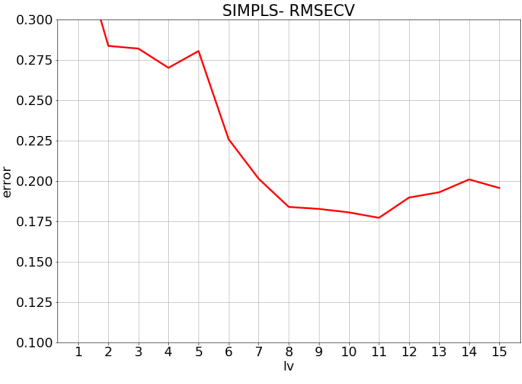

(a)

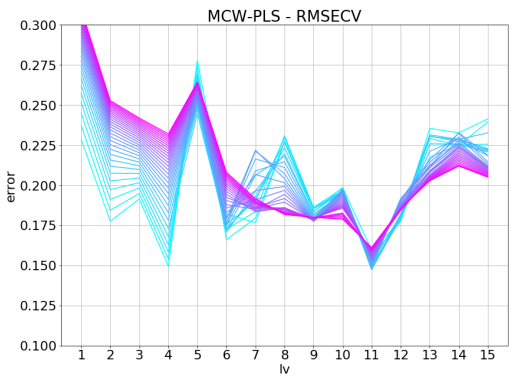

(b)

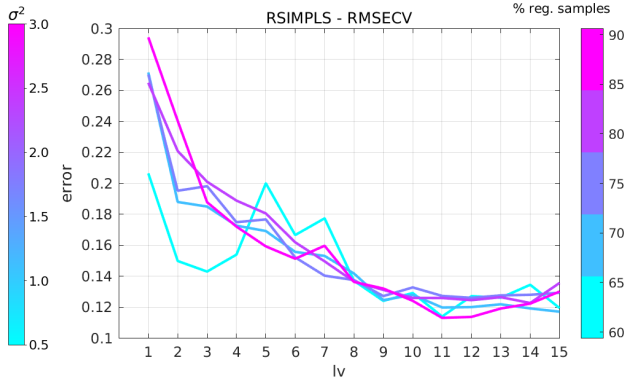

(c)

FIGURE 7 RMSECV curves Corn dataset

this dataset and prediction performance of several calibration algorithms can be found in Mou et al. (2018) 14 .

We focused here on showing the behavior of the RMSECV curves, model performance and comparison of sample weights for predicting the moisture content (\%). Figure 7 shows the RMSECV curves for the three bilinear algorithms. SIMPLS showed a global minimum at 11 latent variables. For RSIMPLS, across the different percentages of regular observations, the global minimum was obtained between 9 and 11 latent variables. This result was better clarified by the resulting RMSECV curves of MCW-PLS, where the global minima were uniformly reached at 11 latent variables. The resemblance between MCW-PLS and RSIMPLS could be observed in the RMSECV curves for low values of $\sigma^{2}$ and percentage of regular observations, where a local minimum was reached at 4 and 3 latent variables, respectively. For MCCR, the optimal RMSECV was found at $\lambda=3 e-07$. In the case of MCW-PLS, we selected a middle value of $\sigma^{2}(1.5)$ because of the uniform RMSECV results at 11 latent variables. This MCW-PLS model rendered a higher prediction performance than SIMPLS and RSIMPLS with the same number of latent variables. MCCR showed the lowest RMSEP but the rather low value of $\lambda$ resulted in a model with large values in the regression coefficients, which impairs the interpretation of the resulting regression signal. However, no other values of $\lambda$ produced satisfactory MCCR models. The results of the selected models are presented in Table 2. The regression coefficients for the MCW-PLS model a the MCCR model are shown in Figure 8.

We compared the sample weights rendered by the best RSIMPLS model with those of the MCW-PLS and MCCR selected models. Figure 9 shows this comparison. A number of samples ranging in the central values of moisture were considered as regular observations by RSIMPLS while being downweighted by MCW-PLS. Likewise, a few samples at the extremes of the moisture values were upweighted by MCWPLS while discarded by RSIMPLS or vice versa. MCCR downweighted to zero three observations and kept almost all the rest of the samples with weights close to 1 .

\subsection{Milk composition dataset: Lactose}

The dataset of milk samples with their Vis/NIR spectra ${ }^{9}$ was used here to calibrate models to predict the lactose content. The spectra of these samples are shown in Figure 3

Figure 10 shows the RMSECV curves for the three bilinear algorithms across the different tuning parameters. The RMSECV curve for SIMPLS reached a minimum at 19 latent variables. For MCW-PLS, the RMSECV curves reached the 


\begin{tabular}{|l|c|c|c|c|c|c|c|c|c|}
\hline \multicolumn{1}{|c|}{ Algorithm } & Model & $\lambda$ & lv & \% reg. samples & $\sigma^{2}$ & RMSECV & R2CV & RMSEP & R2P \\
\hline SIMPLS & 1 & & 11 & & & 0.177 & 0.800 & 0.153 & 0.870 \\
\hline MCW-PLS & 1 & & 11 & & 1.5 & 0.152 & 0.837 & 0.139 & 0.883 \\
\hline & 1 & & 11 & $59 \%$ & & 0.114 & 0.905 & 0.179 & 0.847 \\
& 2 & & 9 & $70 \%$ & & 0.127 & 0.882 & 0.218 & 0.808 \\
RSIMPLS & 3 & & 10 & $80 \%$ & & 0.126 & 0.890 & 0.217 & 0.811 \\
& 4 & & 11 & $91 \%$ & & 0.113 & 0.892 & 0.176 & 0.835 \\
\hline MCCR & 1 & $3 \mathrm{e}-07$ & & & & 0.134 & 0.890 & 0.123 & 0.884 \\
\hline
\end{tabular}

TABLE 2 Selected models for Corn dataset

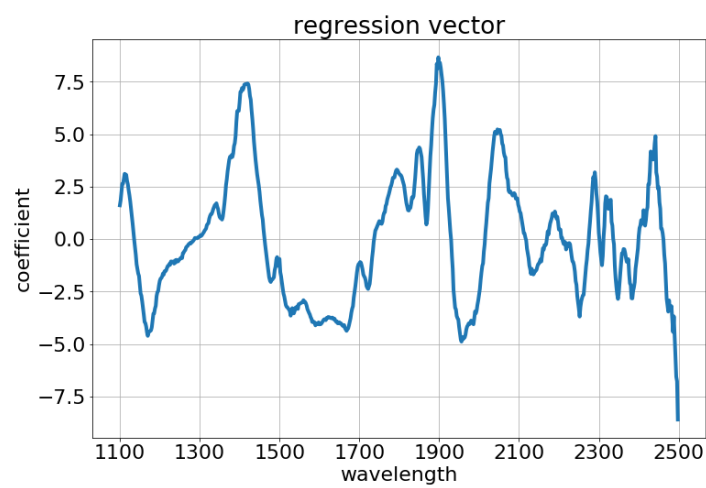

(a) MCW-PLS Model 1

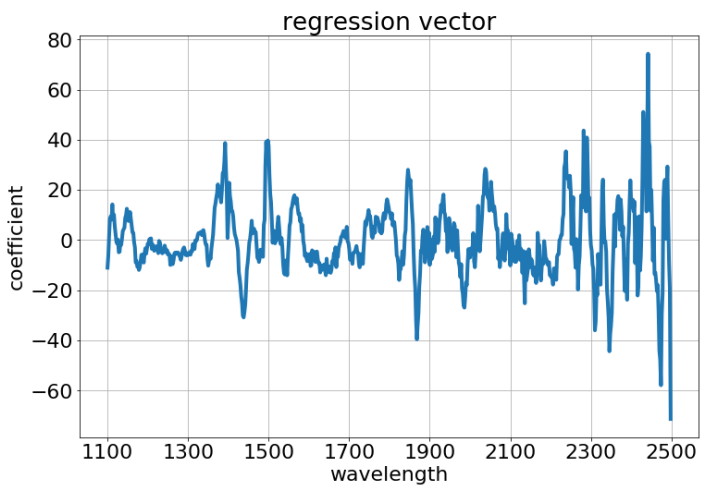

(b) MCCR Model 1

FIGURE 8 Regression vectors MCW-PLS and MCCR Corn dataset (See Table 2)

global minimum at 15 latent variables for $\sigma^{2}$ values lower than 1.5 and at 19 latent variables for values of $\sigma^{2}$ larger than 3. For RSIMPLS, the minimum RMSECV values were in the range 8 to 12 latent variables. The minimum RMSECV value for MCCR was reached at $\lambda=0.0003$. For MCW-PLS and RSIMPLS 2 models were selected to show the performance for a pair

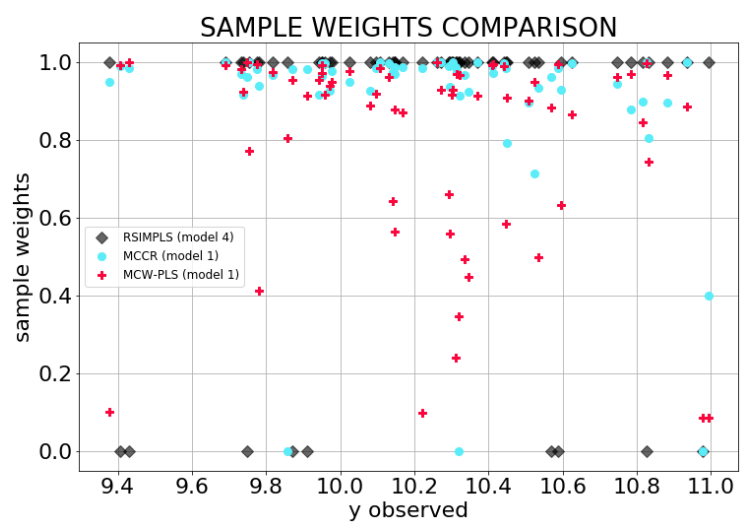

FIGURE 9 Sample weights comparison RSIMPLS, MCCR and MCW-PLS for Corn dataset (See Table 2) of values of the robustness parameter and percentage of regular observations, respectively. The prediction performance of the selected models is shown in Table 3 .

The lowest RMSEP values for the set of selected models were rendered by the MCW-PLS models and SIMPLS. All the models of RSIMPLS had a higher RMSEP. The performance of the selected model for MCCR was similar to that of MCW-PLS and SIMPLS. Nonetheless, MCW-PLS resulted in a model with less latent variables after lowering the value of $\sigma^{2}$.

Motivated by the behavior of the robustness level and the prediction performance of the MCW-PLS calibration models previously presented, the weights of the samples were further analyzed. This was done based on Model 1 for RSIMPLS as it rendered the lowest RMSEP within this algorithm, Model 1 for MCW-PLS as it rendered the closest RMSEP to the minimum observed with a higher level of robustness, and the MCCR model. Figure 11 shows the sample weights comparison. It can be seen that several observations whose weights are somewhere in-between 0 and 1 were discarded by RSIMPLS.

We tested the capacity of MCW-PLS and MCCR to reveal informative samples by selecting subgroups in the calibration set based on the sample weights given by each selected 


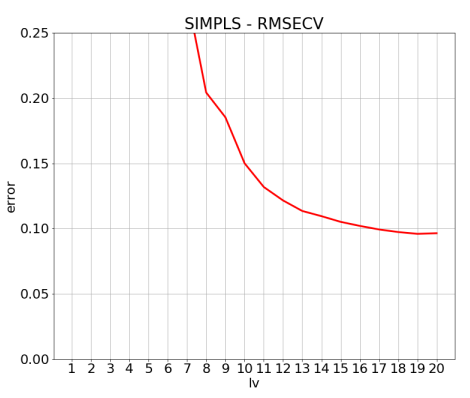

(a)

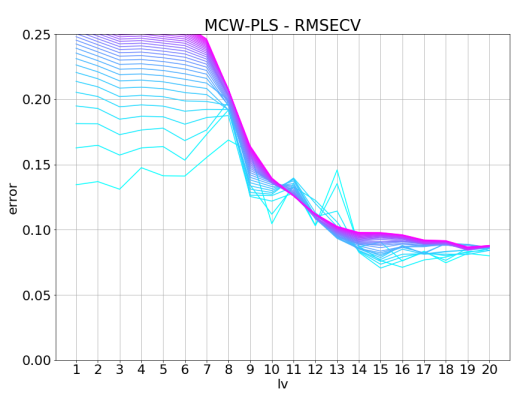

(b)
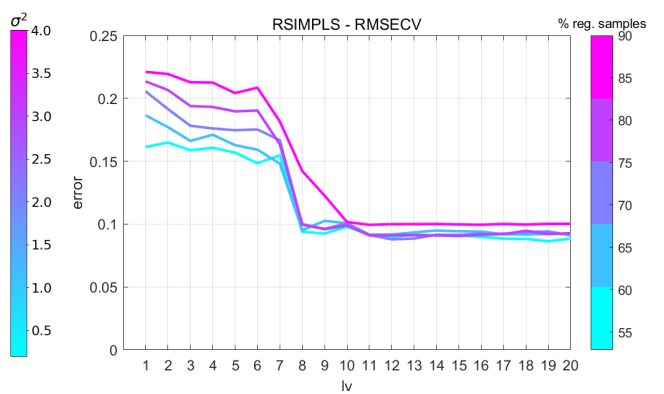

(c)

FIGURE 10 RMSECV curves Milk dataset

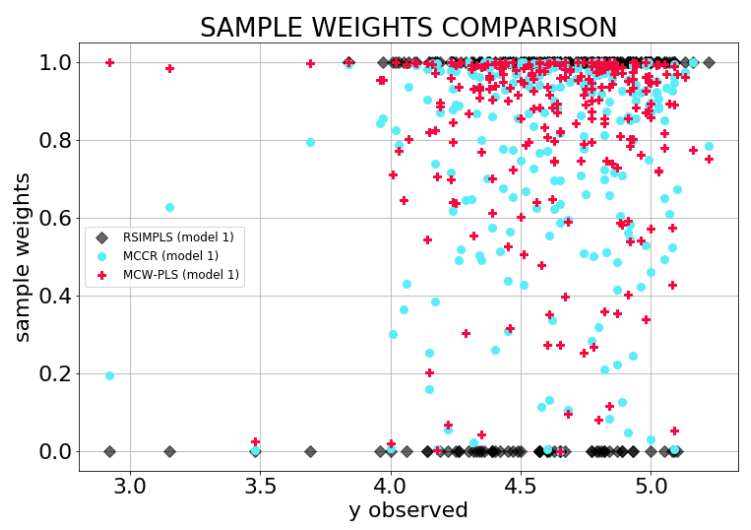

FIGURE 11 Sample weights comparison RSIMPLS, MCCR and MCW-PLS for Milk dataset (See Table 3 )

model of these two algorithms. As the sample weights range between 0 and 1 , subgroups were selected according to $w_{i} \geq$ $0.85,0.9,0.95,0.99$. We stopped at 0.85 because at that point more than $50 \%$ of the samples were included and the RMSEP improved very slowly. When inspecting the RMSECV curves for the models, the long tail behavior in SIMPLS did not provide a global RMSECV minimum. Therefore, the model complexity was kept at 15 and 19 latent variables as suggested by the previous MCW-PLS models. The models were tested on the same test set.

The performance measures for the SIMPLS models based on the MCW-PLS sample weights are shown in Table 4 . Contrary to RSIMPLS, good models could be built with less than $50 \%$ of the observations. In a calibration set of 240 samples, models based on about 100 observations rendered nearly the same prediction performance as that based on the entire calibration set. Table 5 shows the analogous results for SIMPLS models based on samples selected according to the MCCR selected model. It can be seen that the prediction performance of the models based on the MCW-PLS sample weights is better with a lower numbers of calibration samples.

\section{5 | CONCLUSIONS}

In this study, the potential of robustness control in multivariate calibration methods based on MCC was evaluated and compared to RSIMPLS. The proposed algorithm Maximum correntropy weighted PLS (MCW-PLS) proved to be beneficial in terms of prediction performance when the assumption of a normal distribution for the data does not necessarily hold within the domain of bilinear models. It was shown that MCWPLS allows to explore the range of robustness control with respect to SIMPLS and RSIMPLS.

The detected features that make MCW-PLS advantageous within the framework of bilinear models in comparison with SIMPLS and RSIMPLS are: the relaxation of the normal distribution assumption, the capacity to retain fewer observations than RSIMPLS, and the soft sample weights that highlight the importance of samples in terms of the information they contain. The results obtained for the milk dataset confirmed that in a set of 240 samples, less than half were sufficient to capture the most relevant information to predict the lactose content.

Based on the results obtained in this study, it is hypothesized that in applications where many possible sources of variability may influence spectral measurements, it is highly advantageous to be able to retain and weight observations according to the amount of relevant information they contribute in predicting the analytes of interest. Discarding observations flagged as outliers based on the assumption of a normal distribution, as done by RSIMPLS, may harm the prediction performance of the models as shown in the results for the three case studies.

The results of the milk case study highlight important conceptual differences between RSIMPLS and MCW-PLS regarding robustness control. While RSIMPLS is strongly capable of detecting outliers and retaining those regular observations that shape the underlying normal distribution, MCW-PLS is strongly focused on highlighting the points that contain the most valuable information in light of good prediction performance of the bilinear calibration model. 


\begin{tabular}{|l|c|c|c|c|c|c|c|c|c|}
\hline \multicolumn{1}{|c|}{ Algorithm } & Model & $\lambda$ & lv & \% reg. samples & $\sigma^{2}$ & RMSECV & R2CV & RMSEP & R2P \\
\hline SIMPLS & 1 & & 19 & & & 0.096 & 0.922 & 0.085 & 0.893 \\
\hline \multirow{2}{*}{ MCW-PLS } & 1 & & 15 & & 1.5 & 0.083 & 0.941 & 0.094 & 0.868 \\
& 2 & & 19 & & 3 & 0.085 & 0.938 & 0.095 & 0.868 \\
\hline \multirow{2}{*}{ RSIMPLS } & 1 & & 12 & $53 \%$ & & 0.090 & 0.895 & 0.152 & 0.711 \\
& 2 & & 12 & $70 \%$ & & 0.088 & 0.896 & 0.155 & 0.710 \\
\hline MCCR & 1 & 0.0003 & & & & 0.094 & 0.912 & 0.104 & 0.834 \\
\hline
\end{tabular}

TABLE 3 Selected models for Milk dataset

\begin{tabular}{|l|c|c|c|c|c|c|c|}
\hline \multicolumn{1}{|c|}{ Algorithm } & lv & threshold $^{1}$ & ncal & RMSECV & R2CV & RMSEP & R2P \\
\hline \multirow{3}{*}{ SIMPLS } & & 0.99 & 53 & 0.127 & 0.900 & 0.125 & 0.781 \\
& \multirow{2}{*}{15} & 0.95 & 104 & 0.067 & 0.970 & 0.099 & 0.851 \\
& & 0.90 & 135 & 0.056 & 0.975 & 0.100 & 0.848 \\
& & 0.85 & 156 & 0.056 & 0.974 & 0.099 & 0.855 \\
\hline \multirow{3}{*}{ SIMPLS } & \multirow{3}{*}{19} & 0.99 & 53 & 0.112 & 0.922 & 0.116 & 0.811 \\
& & 0.95 & 104 & 0.053 & 0.981 & 0.099 & 0.853 \\
& & 0.90 & 135 & 0.047 & 0.983 & 0.097 & 0.857 \\
& & 0.85 & 156 & 0.048 & 0.981 & 0.098 & 0.856 \\
\hline
\end{tabular}

${ }^{1}$ Minimum value of sample weights to select observations

TABLE 4 SIMPLS models on subsets according to MCW-PLS sample weights from Model 1 in Table 3

Within the framework of maximum correntropy, MCW-PLS may offer a better trade-off between prediction performance and interpretation of the regression coefficients than MCCR. Moreover, MCW-PLS lies in the framework of bilinear models which enable the representation of high-dimensional data in low-dimensional spaces for further statistical and visualization analyses.

\section{1 | Future work}

Tuning the complexity of models based on PLS is not always straightforward. Based on the results shown in this work, we hypothesize that the complexity of the model can be more clearly detected with MCW-PLS than the other two algorithms. We think that the soft weights of samples in MCW-PLS are more vulnerable when changing the model complexity than the hard weights of RSIMPLS. Therefore, it is of interest to study the behavior of the optimal model complexity across the different PLS algorithms based on robustness control.

\section{ACKNOWLEDGMENTS}

Valeria Fonseca Diaz is funded as aspirant to doctoral fellow of the Research Foundation-Flanders (FWO Brussels, Belgium).
We thank professor Peter Goos and Dr. Raffaele Vitale (KU Leuven, Belgium) for their contributions and feedback.

\section{MATERIAL}

All the data, including the public datasets, methods and computational experiments are available on request. The Corn dataset is available at http://www.eigenvector.com/data/Corn/ and the Octane dataset is available within the $\mathrm{R}$ package $\operatorname{rrcov} 23$.

\section{References}

1. Martens Harald. Quantitative Big Data: Where chemometrics can contribute. Journal of Chemometrics. 2015;29(11):563-581.

2. De Jong Sijmen. SIMPLS: An alternative approach to partial least squares regression. Chemometrics and Intelligent Laboratory Systems. 1993;18(3):251-263.

3. Feng Yunlong, Huang Xiaolin, Shi Lei, Yang Yuning, Suykens Johan A K. Learning with the Maximum Correntropy Criterion Induced Losses for Regression. Journal of Machine Learning Research. 2015;16:993-1034. 


\begin{tabular}{|l|c|c|c|c|c|c|c|}
\hline \multicolumn{1}{|c|}{ Algorithm } & lv & threshold $^{1}$ & ncal & RMSECV & R2CV & RMSEP & R2P \\
\hline \multirow{3}{*}{ SIMPLS } & & 0.99 & 38 & 0.080 & 0.944 & 0.164 & 0.619 \\
& \multirow{2}{*}{15} & 0.95 & 82 & 0.046 & 0.974 & 0.125 & 0.762 \\
& & 0.90 & 116 & 0.050 & 0.970 & 0.115 & 0.799 \\
& & 0.85 & 137 & 0.051 & 0.970 & 0.112 & 0.809 \\
\hline \multirow{3}{*}{ SIMPLS } & \multirow{3}{*}{19} & 0.99 & 38 & 0.081 & 0.943 & 0.159 & 0.638 \\
& & 0.95 & 82 & 0.041 & 0.980 & 0.118 & 0.786 \\
& & 0.90 & 116 & 0.043 & 0.977 & 0.109 & 0.819 \\
& & 0.85 & 137 & 0.047 & 0.975 & 0.108 & 0.819 \\
\hline
\end{tabular}

${ }^{1}$ Minimum value of sample weights to select observations

TABLE 5 SIMPLS models on subsets according to MCCR sample weights from Model 1 in Table 3

4. Hubert M., Vanden Branden K.. Robust methods for partial least squares regression. Journal of Chemometrics. 2003;17(10):537-549.

5. Hubert Mia, Rousseeuw Peter J., Van Aelst Stefan. HighBreakdown Robust Multivariate Methods. Statistical Science. 2008;23(1):92-119.

6. Hubert Mia, Rousseeuw Peter J., Vanden Branden Karlien. ROBPCA: A new approach to robust principal component analysis. Technometrics. 2005;47(1):64-79.

7. Fearn Tom, Pérez-Marín Dolores, Garrido-Varo Ana, Guerrero-Ginel José Emilio. A Bayesian framework for near infrared calibration. In: ; 2010.

8. Principe Jose C. Information Theoretic Learning. Renyi's Entropy and Kernel Perspectives. Springer; 2010.

9. Aernouts B., Polshin E., Lammertyn J., Saeys W.. Visible and near-infrared spectroscopic analysis of raw milk for cow health monitoring: Reflectance or transmittance? Journal of Dairy Science. 2011;94(11):5315-5329.

10. Beghi Roberto, Giovenzana Valentina, Brancadoro Lucio, Guidetti Riccardo. Rapid evaluation of grape phytosanitary status directly at the check point station entering the winery by using visible/near infrared spectroscopy. Journal of Food Engineering. 2017;204:46-54.

11. Beghi Roberto, Giovenzana Valentina, Tugnolo Alessio, Guidetti Riccardo. Application of visible/near infrared spectroscopy to quality control of fresh fruits and vegetables in large-scale mass distribution channels: a preliminary test on carrots and tomatoes. Journal of the Science of Food and Agriculture. 2018;98(7):2729-2734.

12. Ran He, Bao-Gang Hu, Wei-Shi Zheng, Xiang-Wei Kong . Robust Principal Component Analysis Based on Maximum Correntropy Criterion. IEEE Transactions on Image Processing. 2011;20(6):1485-1494.
13. Peng Jiangtao, Guo Lu, Hu Yong, Rao Kai Feng, Xie Qiwei. Maximum correntropy criterion based regression for multivariate calibration. Chemometrics and Intelligent Laboratory Systems. 2017;161(June 2016):27-33.

14. Mou Yi, Zhou Long, Chen Weizhen, Fan Jijun, Zhao $\mathrm{Xu}$. Maximum correntropy criterion partial least squares. Optik. 2018;165:137-147.

15. Naes Tormod, Martens Harald. Comparison of Prediction Methods for Multicollinear Data. Communications in Statistics - Simulation and Computation. 1985;14(3):545576.

16. Hubert Mia, Debruyne Michiel. Minimum covariance determinant. Wiley Interdisciplinary Reviews: Computational Statistics. 2010;2(1):36-43.

17. Yuan Xiao Tong, Hu Bao Gang. Robust feature extraction via information theoretic learning. Proceedings of the 26th International Conference On Machine Learning, ICML 2009. 2009;:1193-1200.

18. Hoerl Arthur E., Kennard Robert W.. Ridge Regression: Biased Estimation for Nonorthogonal Problems. Technometrics. 1970;12(1):55-67.

19. Ramirez-Lopez Leonardo, Schmidt Karsten, Behrens Thorsten, Wesemael Bas, Demattê Jose A.M., Scholten Thomas. Sampling optimal calibration sets in soil infrared spectroscopy. Geoderma. 2014;226-227(1):140-150.

20. Pedregosa F., Varoquaux G., Gramfort A., et al. Scikitlearn: Machine Learning in Python. Journal of Machine Learning Research. 2011;12:2825-2830.

21. Verboven Sabine, Hubert Mia. LIBRA: a MATLAB Library for Robust Analysis. Chemometrics and Intelligent Laboratory Systems. 2005;75:127-136. 
22. Hubert Mia, Verboven Sabine. A robust PCR method for high-dimensional regressors. Journal of Chemometrics. 2003;17(8-9):438-452.

23. Todorov Valentin, Filzmoser Peter. An Object-Oriented Framework for Robust Multivariate Analysis. Journal of Statistical Software. 2009;32(3):1-47.

How to cite this article: Valeria Fonseca Diaz, Bart De Ketelaere, and Wouter Saeys (), Robustness control in bilinear modeling based on maximum correntropy, Journal of Chemometrics, ;: . 